Article

\title{
On the Outage Capacity of Transdermal Optical Wireless Links with Stochastic Spatial Jitter and Skin-Induced Attenuation
}

\author{
George K. Varotsos ${ }^{1}$ (D), Konstantinos Aidinis ${ }^{2,3}$ and Hector E. Nistazakis ${ }^{1, *(\mathbb{D})}$ \\ 1 Section of Electronic Physics and Systems, Department of Physics, National and Kapodistrian University of \\ Athens, 15784 Athens, Greece; georgevar@phys.uoa.gr \\ 2 Department of Electrical and Computer Engineering, Ajman University, Ajman P.O. Box 346, \\ United Arab Emirates; k.aidinis@ajman.ac.ae \\ 3 Centre of Medical and Bio-Allied Health Sciences Research (CMBHSR), Ajman University, \\ Ajman P.O. Box 346, United Arab Emirates \\ * Correspondence: enistaz@phys.uoa.gr; Tel.: +30-210-7276710
}

check for

updates

Citation: Varotsos, G.K.; Aidinis, K.; Nistazakis, H.E. On the Outage Capacity of Transdermal Optical Wireless Links with Stochastic Spatial Jitter and Skin-Induced Attenuation. Photonics 2021, 8, 553. https:// doi.org/10.3390/photonics 8120553

Received: 29 September 2021 Accepted: 30 November 2021 Published: 3 December 2021

Publisher's Note: MDPI stays neutral with regard to jurisdictional claims in published maps and institutional affiliations.

Copyright: (c) 2021 by the authors. Licensee MDPI, Basel, Switzerland. This article is an open access article distributed under the terms and conditions of the Creative Commons Attribution (CC BY) license (https:// creativecommons.org/licenses/by/ $4.0 /)$.

\begin{abstract}
The tremendous development of both optical wireless communications (OWC) and implantable medical devices (IMDs) has recently enabled the establishment of transdermal optical wireless (TOW) links that utilize light waves to transfer information inside the living body to the outside world and conversely. Indeed, numerous emerging medical applications such as cortical recording and telemetry with cochlear implants require extremely high data rates along with low power consumption that only this new technology could accommodate. Thus, in this paper, a typical TOW link is investigated in terms of outage capacity which is a critical performance metric that has so far not been evaluated for such wireless systems in the open technical literature. More precisely, an outage capacity analysis is performed considering both skin-induced attenuation and stochastic spatial jitter, i.e., pointing error effects. Analytical expressions and results for the outage capacity are derived for a variety of skin channel conditions along with varying stochastic pointing errors which demonstrate the feasibility of this cross-field cooperation. Lastly, the corresponding simulation outcomes further validate our suggestions.
\end{abstract}

Keywords: optical wireless communications (OWC); transdermal optical wireless (TOW) links; implantable medical devices (IMDs); outage capacity; pointing errors

\section{Introduction}

TOW links are rapidly gaining popularity as a very promising alternative to conventional transdermal radiofrequency (RF) wireless links, which are commonly used todayfor varying medical applications [1-3]. Transdermal RF has been shown to support data rates of some Mbps up to $24 \mathrm{Mbps}$, consuming at the same time at least $30 \mathrm{~mW}$ [4]. However, many emerging medical applications such as recording neural signals from in-body devices and actuating these devices from out-of-body signals to guide a prosthesis, require even higher capacities to emulate similar performance to that of human organs, such as the cochlea, along with lower power consumption [5-10]. Another representative example ofthe need forhigher-speed bidirectional transdermal communication than RF is when neural signals are recorded, sampled, processed, and used to actuate artificial limbs for rehabilitation human affected by paralysis resulting from stroke, head injury, spinal cord injury, and other neurological disorders. This process can relieve pain, improve neurological disorder, or even regain functionality of damaged limbs [11]. Additionally, in comparison with the achievable data rates via transcutaneous $\mathrm{RF}$, even higher-speed transdermal wireless links may be needed for such brain-machine interface applications [12-15]. In fact, $50 \mathrm{Mbps}$ would be required when 100 channels are simultaneously recorded [1].

As is the case with the wider free-air optical wireless communication systems [16-18], according to TOW modality, the data aretransmitted via a very-high-frequency light carrier 
and, therefore, with an extremely high data rate which can meet the above growing capacity and energy consumption demands [19]. Nevertheless, owing to the different channel properties of human skin in comparison with atmospheric channels, the utilized wavelength window for TOW is between $600 \mathrm{~nm}$ and $1300 \mathrm{~nm}$, while typical TOW link lengths are limited up to a few millimeters due to skin-induced attenuation and transdermal pathloss [20-23]. Another but equally significant advantage of TOW versus RF transdermal modality is that the former ensures that there is no interference with existing RF networks or electronic equipment, while the latter suffers from electromagnetic interference (EMI) and multipath fading within a highly regulated spectrum [24-26].

In view of the above, TOW links have attracted particular research interest in the last few years. The feasibility of establishing TOW links has been experimentally validated by different research organizations around the world $[2,5,12,13,27-33]$. In short, the feasibility of establishing modulated retroreflective and direct TOW links was demonstrated in $[5,30]$, while, in [33], a bidirectional TOW link system for artificial hearts was employed. In [28], a $75 \mathrm{Mbps}$ invitro TOW link was presented, while the potential of establishing in-vivo TOW links was verified in [29] by achieving data rates up to $100 \mathrm{Mbps}$ with $2.1 \mathrm{~mW}$ of electrical power consumption. Moreover, the feasibility of establishing a bidirectional TOW link for brain-machine interface was validated in [12,13]. Nevertheless, what all these papers have in common is that pointing errors, which refer to unavoidable, random misalignments between transmitter and receiver terminals and may result in significant TOW performance degradation, were either neglected or considered as a deterministic effect. The concept of the stochastic nature of pointing errors was first modeled and reported in [34] and thenin [9,35]. Even more recently, the stochastic impact of pointing errors was reported in $[3,10,19,24,36-40]$. In these papers, considering the joint impact of transdermal pathloss and stochastic pointing errors, the TOW performance was evaluated in terms of outage probability, average signal-to-noise ratio (SNR), average ergodic capacity, average bit error rate (ABER), or average symbol error probability (ASEP) for various TOW system realizations.

Another critical performance metric, especially for high-speed communication systems is the outage capacity, which denotes the maximum data transmission rate of reliable communications under a specific outage probability [41,42]. However, to the best of our knowledge, although outage capacity has been extensively utilized for the wider free air communication systems [16,41-45], it has not yet been reported in the TOW technical literature. Motivated by the above, in the current contribution, the outage capacity for typical TOW link configurations is estimated, in the presence of skin-induced attenuation and stochastic pointing errors. Under these circumstances, analytical outage capacity expressions are derived with their corresponding results verifying the accuracy of our proposed analysis over a wide average electrical SNR range.

\section{System and Channel Model}

The TOW under investigation mainly consists of three fundamental parts: the outof-body transmitter unit, the skin propagation medium which acts as the communication channel, and the in-body receiver unit. The external transmitter emits via a laser source the information-bearing light carrier which is modulated with on off keying (OOK) modulation format. After traversingthe propagation medium, the internal unit collects the light signal via an appropriate photodiode, and then the received signal is demodulated in order to provide the appropriate stimulations, after being processed by the internal digital signal processing unit and the stimulation unit. Under these circumstances, the received signal is expressed as follows [35]:

$$
y=\eta h x+n,
$$

where $\eta$ represents the photo-current conversion ratio at the receiver side, $h$ is the skin channel state, $x$ stands for the modulated binary transmitted signal, and $n$ denotes the additive noise described as a zero mean complex Gaussian process with variance $\sigma^{2}$ [36]. 
The channel state is expressed asfollows [10]:

$$
h=h_{l} h_{p},
$$

where $h_{l}$ represents the deterministic channel coefficient owing to transdermal propagation loss, and $h_{p}$ is the stochastic process that describes the misalignment-induced geometric spread at the receiver aperture due to pointing errors [35].

The deterministic pathloss parameter of Equation (2) is given as follows [34]:

$$
h_{l}=\exp \left[-\frac{1}{2} \alpha(\lambda) \delta\right],
$$

where $\delta$ is the dermal thickness of the skin channel which practically translates into the transdermal link length, and $\alpha(\lambda)$ is the corresponding attenuation coefficient which depends strongly on the utilized optical wavelength, $\lambda$. Indeed, within the wavelength region between $400 \mathrm{~nm}$ and $1800 \mathrm{~nm}$, the latter coefficient is expressed asfollows [9]:

$$
\alpha(\lambda)=\sum_{i=1}^{8} a_{i} \exp \left[-\left(\frac{\lambda-b_{i}}{c_{i}}\right)^{2}\right],
$$

where wavelength values are expressed in $\mathrm{nm}$, and the remainingparameter values are obtained as described below [24,35].

In fact, for TOW links, we focus on their operation at wavelengths between $600 \mathrm{~nm}$ and $1300 \mathrm{~nm}$, since, within this wavelength region, known as the medical or tissue optical window, the photon absorption that stems mainly from water content or hemoglobin and melanin can be minimized [3,23]. Optical signals with smaller or larger wavelengths are practically inappropriate for the establishment of TOW links due to this extremely strong skin-induced photon absorption.

In addition to the selected optical wavelength, the value of the skin attenuation coefficient depends heavily on the optical properties of the skin and can be obtained numerically from experimental results [20]. On the basis of these values and by employing the trust region method, the latter expression along with its parameter values described in Table 1 can be obtained. It is notable that the accuracy of the latter expression was estimated to be higher than $99.7 \%$ by using the coefficient of determination ( $R$-squared), which is the square of the correlation between the response values and the predicted response values and can be expressed as the ratio of the sum of squares of the regression and the total sum of squares [9].

Table 1. The $a_{i}, b_{i}$, and $c_{i}$ parameter values.

\begin{tabular}{cccc}
\hline $\boldsymbol{i}$ & $\boldsymbol{a}_{\boldsymbol{i}}$ & $\boldsymbol{b}_{\boldsymbol{i}}$ & $\boldsymbol{c}_{\boldsymbol{i}}$ \\
\hline 1 & 10 & 0.35 & 0.065 \\
2 & 4.5 & 0.42 & 0.25 \\
3 & 13.48 & -1.5 & 50.12 \\
4 & 14.7 & 1442 & 49.35 \\
5 & 7.435 & 1499 & 75.88 \\
6 & 48 & 3322 & 1033 \\
7 & 594.1 & -183 & 285.9 \\
8 & 11.47 & -618.5 & 1054 \\
\hline
\end{tabular}

Additionally, the random variable that describes stochastic pointing errors obtains the following probability density function (PDF) $[16,39,43]$ :

$$
f_{h_{p}}\left(h_{p}\right)=\psi^{2} A_{0}^{-\psi^{2}} h_{p}^{\psi^{2}-1}, 0 \leq h_{p} \leq A_{0},
$$


where $\psi=w_{e q} / 2 \sigma$ is proportional to the amount of pointing error strength with $w_{e q}$ denoting the equivalent beam radius in the detector aperture and $\sigma$ representing the spatial jitter, i.e., the pointing error displacement. Specifically, larger $\psi$ parameter values imply stronger pointing errors and, thus, more severe misalignment-induced fading. Indeed, increased $\psi$ values practically refer to smaller spatial jitter values, which means that the beam footprint overlaps the detector aperture to a greater extent, thus describing smaller amounts of pointing mismatch. Consequently, when $\psi \rightarrow \infty$ or practically when $\psi$ obtains very large values, pointing errors can be considered as a negligible effect. Furthermore $w_{\text {eq }}=\left[\frac{\sqrt{\pi} \operatorname{erf}(v) w_{\delta}^{2}}{2 \operatorname{vexp}\left(-v^{2}\right)}\right]^{1 / 2}$, where erf(.) stands for the error function, (Equation (8.250.1) in [46]), and $v=\frac{\sqrt{\pi} \rho}{\sqrt{2} w_{\delta}}$, where $\rho$ represents the radius of the circular receiver aperture and $A_{0}=\operatorname{erf}^{2}(v)$ isthe fraction of the collected power at $\rho=0[16,24]$. Additionally, $w_{\delta}$, which represents the corresponding beam waist on the receiver plane at a propagating transdermal distance $\delta$ along with a transmitter divergence angle $\theta$, is obtained as follows [10,38]:

$$
w_{\delta}=\delta \tan (\theta / 2)
$$

By using Equations (2) and (5), the PDF of the random variable $h$ can be obtained asfollows [16,19]:

$$
f_{h}(h)=\psi^{2} A_{0}^{-\psi^{2}} h_{l}^{-\psi^{2}} h^{\psi^{2}-1}, 0 \leq h \leq A_{0} h_{l} .
$$

Moreover, by using Equations (2) and (3), the instantaneous electrical SNR, $\gamma=\eta^{2} h^{2} P_{s} N_{0}^{-1}$, is expressed asfollows:

$$
\gamma=\eta^{2} h_{l}^{2} h_{p}^{2} P_{s} N_{0}^{-1}=\eta^{2} h_{p}^{2} \exp [-\alpha(\lambda) \delta] P_{s} N_{0}^{-1},
$$

where $P_{S}$ and $N_{0}$ represent the signal and noise optical power spectral density (PSD), respectively [34].

Consequently, the average electrical SNR, $\mu$, is obtained asfollows [35]:

$$
\mu=\frac{(\eta E[h])^{2} P_{s}}{N_{0}}=\frac{\eta^{2} \psi^{2} A_{0}^{2} P_{s} \exp [-\alpha(\lambda) \delta]}{\left(\psi^{2}+2\right) N_{0}}
$$

where $E[$.$] denotes expectation.$

Next, by using Equations (7) and (8), along with applying the standard technique of transforming random variables $f_{\gamma}(\gamma)=\frac{f_{h}(h)}{|\partial \gamma / \partial h|} \mid h=\sqrt{\frac{\gamma}{\eta^{2} P_{s} N_{0}^{-1}}}$ [47], the PDF of random variable $\gamma$ is obtained asfollows:

$$
f_{\gamma}(\gamma)=\frac{\psi^{2}}{2} A_{0}^{-\psi^{2}} h_{l}^{-\psi^{2}}\left(\eta^{2} N_{0}^{-1} P_{S}\right)^{\frac{-\psi^{2}}{2}} \gamma^{\frac{\psi^{2}-2}{2}}, 0 \leq \gamma \leq A_{0}^{2} h_{l}^{2} \eta^{2} N_{0}^{-1} P_{S} .
$$

By substituting Equation (9) into Equation (10), we get

$$
f_{\gamma}(\gamma)=\frac{\psi^{2}}{2}\left(\frac{\psi^{2}}{\psi^{2}+2}\right)^{\frac{\psi^{2}}{2}} \mu^{\frac{-\psi^{2}}{2}} \gamma^{\frac{\psi^{2}-2}{2}}, 0 \leq \gamma \leq A_{0}^{2} h_{l}^{2} \eta^{2} N_{0}^{-1} P_{S} .
$$

\section{Outage Capacity}

A very critical performance metric for each OWC system is its outage capacity, $C_{\text {out }}$, which stands for the capacity guaranteed for a percentage rate of $(1-r)$ of the channel realizations [44,45].

$$
\operatorname{Pr}\left[C<C_{\text {out }}\right]=r,
$$


where $\operatorname{Pr}[$.$] denotes probability, and C$ represents the instantaneous channel capacity which is obtained as follows [43]:

$$
C=B \log _{2}(1+\gamma)=\frac{1}{\ln 2} B \ln (1+\gamma),
$$

where $B$ is the channel's bandwidth.

It is appropriate to clarify here the differences between outage probability, outage capacity, and capacity metrics. Capacity denotes the achievable maximum data rate just only for a specific moment, while the outage capacity represents the capacity guaranteed for a specific probability of the channel realizations. Additionally, the outage probability represents the probability that the instantaneous SNR at the receiver side falls below a specific SNR threshold that corresponds to the receiver's sensitivity, while the outage capacity could represent the capacity guaranteed for a specific outage probability. Moreover, higher outage probability values correspond to a degraded outage performance for the system, while higher outage capacity values correspond to an upgraded outage performance for the system [36,44]. Indeed, considering Equation (12), the outage capacity refers to the minimum capacity which is required for a specificpercentage rate of channel realizations, while outage probability refers to the required instantaneous SNR threshold.

Since, in a real wireless channel, the instantaneous electrical SNR, $\gamma$, is a random variable, it becomes evident from Equation (13) that $C$ is also a random variable. Therefore, the probability of Equation (12) is evaluated asfollows [42,45]:

$$
r=\int_{0}^{C_{\text {out }}} f_{C}(C) d C
$$

where $f_{C}(C)$ is the PDF of the random variable $C$.

Using Equations (11) and (13), along with standard technique of transforming random variables, $f_{C}(C)=\frac{f_{\gamma}(\gamma)}{|\partial C / \partial \gamma|} \mid \gamma=2^{\frac{C}{B}}-1$ [47], the latter PDF is obtained asfollows:

$$
f_{C}(C)=\frac{\psi^{2} \ln 2}{2 B}\left(\frac{\psi^{2}}{\psi^{2}+2}\right)^{\frac{\psi^{2}}{2}} \mu^{\frac{-\psi^{2}}{2}} 2^{C / B}\left(2^{C / B}-1\right)^{\frac{\psi^{2}-2}{2}}
$$

Thus, by substituting Equation (15) into Equation (14), we get

$$
r=\frac{\psi^{2} \ln 2}{2 B}\left(\frac{\psi^{2}}{\psi^{2}+2}\right)^{\frac{\psi^{2}}{2}} \mu^{\frac{-\psi^{2}}{2}} \int_{0}^{C_{\text {out }}} 2^{C / B}\left(2^{C / B}-1\right)^{\frac{\psi^{2}-2}{2}} d C
$$

In order to calculate the integral of Equation (16), we set $u=\sqrt{2^{C / B}-1}, d u=$ $\frac{2^{C / B} \ln 2}{2 B \sqrt{2^{C / B}-1}} d C$. Thus, the above integral is calculated as

$$
\int_{0}^{\sqrt{{ }^{C_{\text {out }} / B}-1}} u^{\psi^{2}-2} u(2 B / \ln 2) d u=\frac{2 B}{\psi^{2} \ln 2}\left(\sqrt{2^{C_{\text {out }} / B}-1}\right)^{\psi^{2}} .
$$

Therefore, by substituting Equation (17) into Equation (16), we obtain

$$
r=\left(\frac{\psi^{2}}{\psi^{2}+2}\right)^{\frac{\psi^{2}}{2}} \mu^{\frac{-\psi^{2}}{2}}\left(\sqrt{2^{C_{\text {out }} / B}-1}\right)^{\psi^{2}}
$$


which can also be expressed by substituting Equation (9) into Equation (18) asfollows:

$$
C_{\text {out }} / B=\log _{2}\left\{10^{\frac{2}{\psi^{2}} \log \left[r\left(\frac{\psi^{2}}{\psi^{2}+2}\right)^{-\frac{\psi^{2}}{2}}\left(\frac{\eta^{2} \psi^{2} A_{0}^{2} P_{s} \exp [-\alpha(\lambda) \delta]}{\left(\psi^{2}+2\right) N_{0}}\right)^{\frac{\psi^{2}}{2}}\right]}+1\right\} .
$$

The derived analytical expressions in Equations (18) and (19) reveal that, in addition to the available bandwidth, the outage capacity for TOW links depends strongly on the pointing error's strength, average electrical SNR at the receiver's side, skin-induced attenuation, and specific link characteristics such as skin channel thickness, power and noise spectral densities, and operational wavelength.

\section{Analytical Results}

In this section, analytical results are graphically depicted that arise from the derived analytical expressions in Equations (18) and (19). Their validity is further verified by appropriate simulations. The diameter of the receiver's aperture, $\rho$, wasset to $0.5 \mathrm{~mm}$, while the operational wavelength, $\lambda$, wasfixed at $1100 \mathrm{~nm}$, which can drastically address skininduced attenuation due to photon absorption, as reported in [9]. Moreover, although the probability $r$ could take numerous values, the values 0.01 and 0.1 werehere utilized, which are very common for any OWC link [42]. Furthermore, $\eta=0.8, \theta=20^{\circ}, P_{s}=1 \mu \mathrm{W} / \mathrm{MHz}$, and $N_{0}=(1.3 \mathrm{pA} / \sqrt{\mathrm{Hz}})^{2}$ [48]. Regarding pointing mismatch, varying weak to strong pointing error effects wereevaluated for two different typical transdermal link lengths, i.e., $(\delta, \sigma / \rho, \psi)=(4 \mathrm{~mm}, 1.1,2.4),(\delta, \sigma / \rho, \psi)=(4 \mathrm{~mm}, 1.5,1.76),(\delta, \sigma / \rho, \psi)=$ $(4 \mathrm{~mm}, 2.0,1.32),(\delta, \sigma / \rho, \psi)=(4 \mathrm{~mm}, 2.3,1.15),(\delta, \sigma / \rho, \psi)=(5 \mathrm{~mm}, 1.1,2.98)$, $(\delta, \sigma / \rho, \psi)=(5 \mathrm{~mm}, 1.5,2.19),(\delta, \sigma / \rho, \psi)=(5 \mathrm{~mm}, 2.0,1.64)$, and $(\delta, \sigma / \rho, \psi)=$ $(5 \mathrm{~mm}, 2.3,1.43)$. Note that $\psi$ values are getting smaller as $\sigma / \rho$ values are getting larger, especially for smaller $\delta$ values, i.e., pointing errors are getting stronger as spatial jitter increases, as expected, especially for shorter transdermal link lengths, according to Equation (6). Indeed, the latter is valid assuming fixed $\theta$ and $\rho$ parameter values as mentioned above. Under these conditions, different typical TOW link configurations wereinvestigated by means of outage capacity over a wide average SNR range, from $0 \mathrm{~dB}$ to $30 \mathrm{~dB}$.

Figure 1 illustrates the outage capacity dependence on the average electrical SNR evolution for a typical TOW link length of $5 \mathrm{~mm}$ with different values of normalized jitter standard deviation, provided that $r=0.1$. We observe that higher jitter values lead to lower $\psi$ parameter values and, thus, to stronger pointing error effects. In fact, as pointing errors effects are getting stronger, significantly lower outage capacity values are depicted, especially for lower average electrical SNR values.

Figure 2 depicts the corresponding analytical results tothose of Figure 1 but with $r=0.01$, i.e., with a smaller value of the probability $r$. Consequently, although similar qualitative results wereobtained for the outage capacity in both cases, the decrease inthe probability value mentioned above broughtabout smaller corresponding outage capacity values. Therefore, the performance comparison between these two figures highlights the outage capacity degradation due to lower $r$ values. This behavior is in good agreement with what happens in the wider OWC field, much less for the same probability values [42]. Additionally, the impact of pointing errors is depicted to become even more severe by decreasing this probability value, especially for lower average electrical SNR values. 


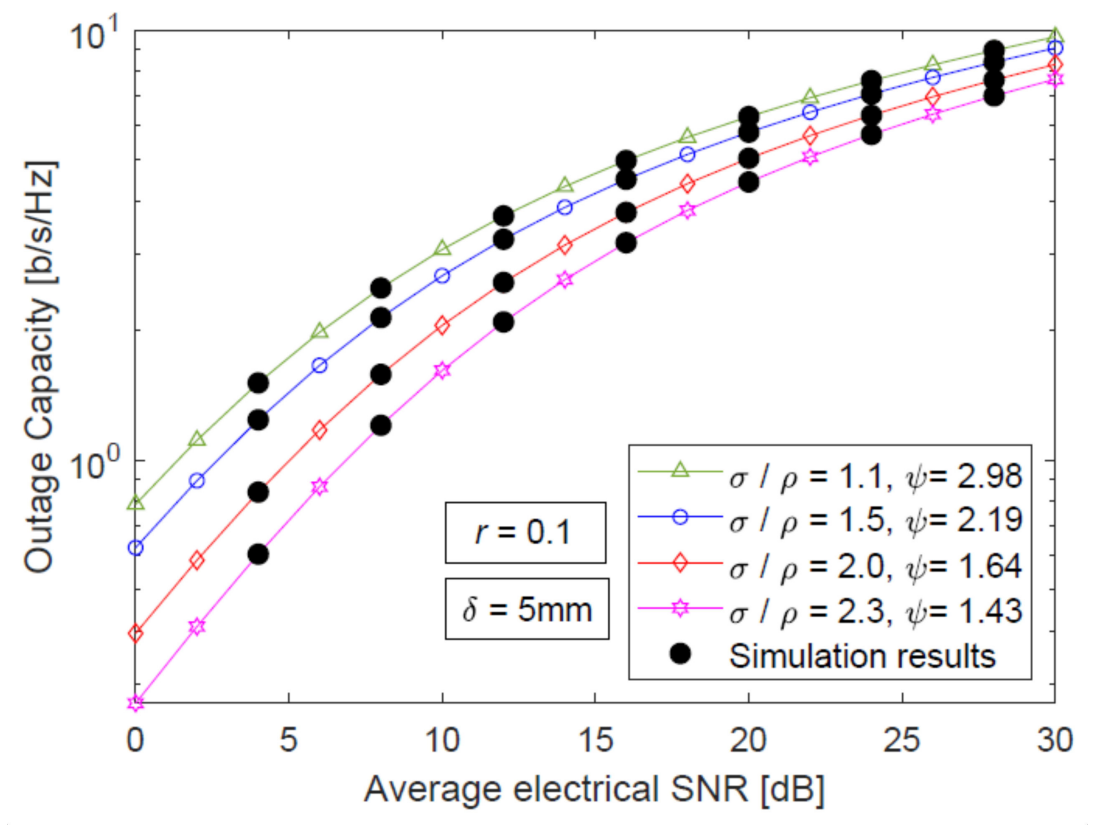

Figure 1. Outage capacity versus average electrical SNR for $\delta=5 \mathrm{~mm}$ and $r=0.1$ under the presence of varying, weak to strong stochastic pointing errors.

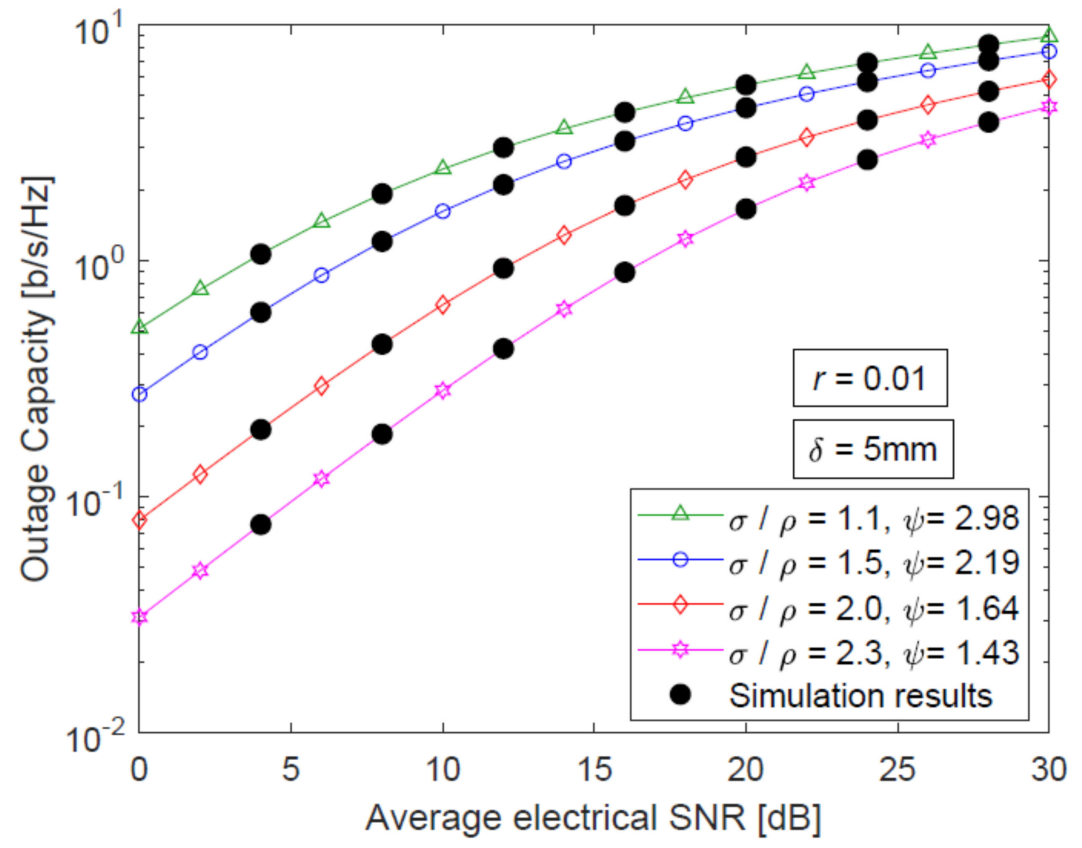

Figure 2. Outage capacity versus average electrical SNR for $\delta=5 \mathrm{~mm}$ and $r=0.01$ under the presence of varying, weak to strong stochastic pointing errors.

Figure 3 presents the corresponding analytical results tothose of Figure 2 but through a shorter transdermal link, i.e., a smaller value of parameter $\delta$. As expected from Equation (6), shorter transdermal link distances led to larger beam waist values for a specific initial divergence angle, which resulted, in turn, in a larger amount of pointing mismatch. Indeed, for the same normalized jitter standard deviation values, we now obtained smaller corresponding $\psi$ parameter values due to the shorter transdermal propagation distance. This translated into more important outage capacity performance degradations due to the presence of stronger pointing errors, especially for lower average electrical SNR values. In this context, Figure 3 highlights the dependence of stochastic pointing error effects 
on transdermal link length, in terms of TOW outage capacity. Note that this behavior is consistent with what happens with other critical TOW outage performance metrics, such as the outage probability and the average bit error rate [35,37]. It should be noted, however, that, for longer TOW distances, i.e., above some additional millimeters, the impact of skininduced attenuation dominates the impact of pointing errors. Thus, after some transdermal propagation distance, the corresponding outage performance will be degraded due to the total transdermal pathloss, despite the increase inbeam waist.

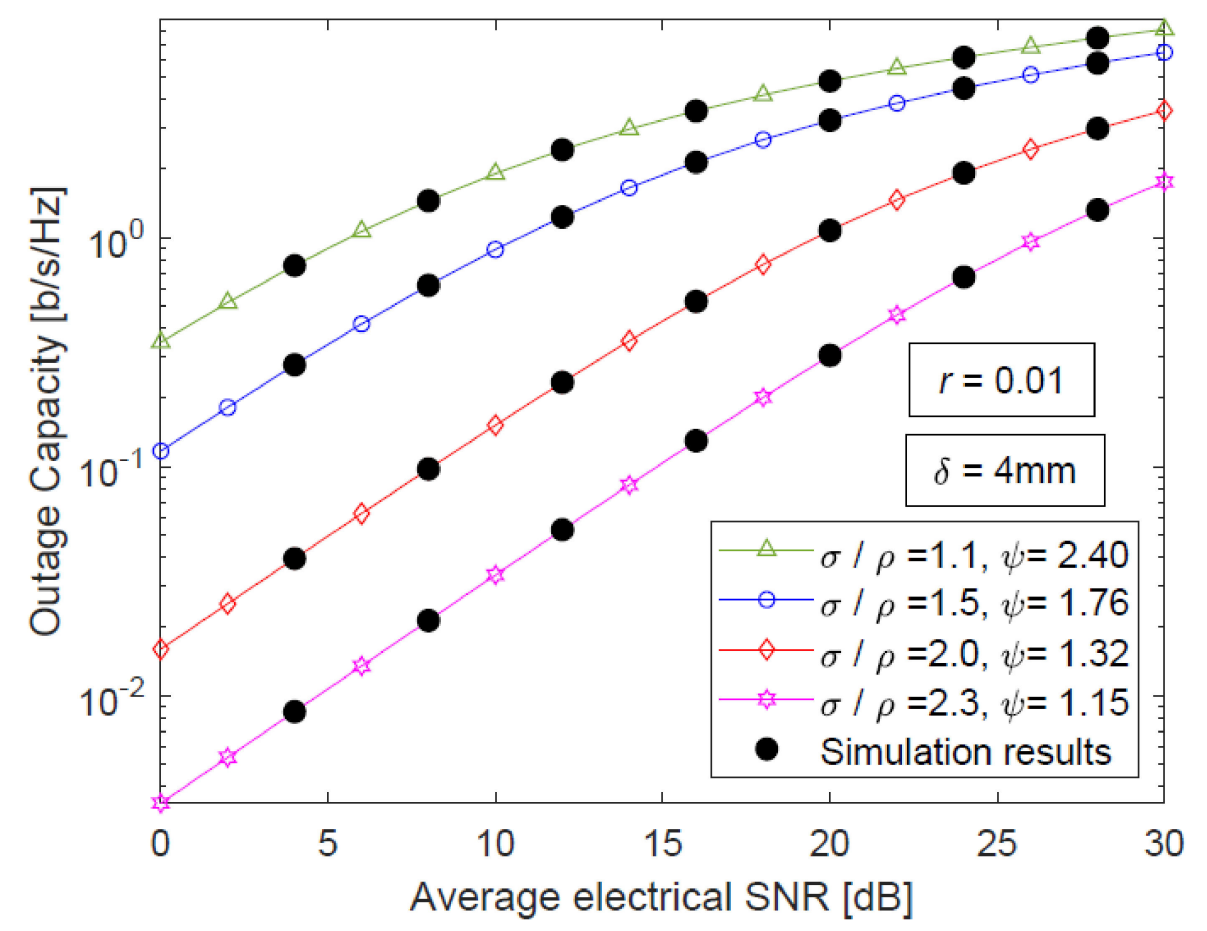

Figure 3. Outage capacity versus average electrical SNR for $\delta=4 \mathrm{~mm}$ and $r=0.01$ under the presence of varying, weak to strong stochastic pointing errors.

\section{Discussion and Conclusions}

In this work, we first investigated the outage capacity for the performance of TOW links with slow-fading skinchannels along with the presence of varying stochastic spatial jitter. In this respect, an outage capacity analysis was performed, while novel analytical expressions were derived for this crucial performance metric which incorporate the most significant parameters and effects that play a key role in TOW performance and availability. Their analytical results which were validated by proper simulations reveal that the achievable TOW outage capacity largely depends on the average electrical SNR at the receiver's side, as well as on stochastic pointing errors' strength, along with transdermal propagating distance for the information-bearing light which penetrates into the skin channel. In this context, the feasibility of establishing high-speed TOW links was demonstrated for typical transdermal link lengths, even in very harsh pointing mismatch conditions. Toward the development of future higher-speed TOW links of enhanced robustness, the analysis proposed can be added to the engineer's quiver as a useful tool for the design of such effective transdermal wireless systems.

In view of the above, the key contribution of this work was to develop a theoretical framework for the outage capacity estimation of typical TOW links quantifying the impact for weak to strong stochastic pointing errors.It should be noted, however, that, for a real human skin channel, human body temperature, in addition to other specific skin and tissue particularities, should be taken into consideration. Nevertheless, the performance comparison ofour findings in TOW area withcorresponding outage capacity results in 
traditional free-air communication systems evaluated in $[42,43]$ reveal the feasibility of our findings and suggestions.

Author Contributions: Conceptualization, G.K.V., K.A. and H.E.N.; methodology, G.K.V., K.A. and H.E.N.; software, G.K.V. and K.A.; validation, G.K.V., H.E.N. and K.A.; investigation, G.K.V., H.E.N. and K.A.; resources, G.K.V., K.A. and H.E.N.; writing—original draft preparation, G.K.V.; writing-review and editing, G.K.V. and H.E.N.; supervision, G.K.V., K.A. and H.E.N.; funding acquisition, G.K.V., K.A. and H.E.N. All authors read and agreed to the published version of the manuscript.

Funding: This research was funded by Ajman University, grant number 2020-IRG-ENIT-02.

Institutional Review Board Statement: Not applicable.

Informed Consent Statement: Not applicable.

Data Availability Statement: Not applicable.

Acknowledgments: The authors acknowledge funding from Ajman University under grant agreement 2020-IRG-ENIT-02.

Conflicts of Interest: The authors declare no conflict of interest.

\section{References}

1. Liu, T.; Bihr, U.; Anis, S.M.; Ortmanns, M. Optical transcutaneous link for low power, high data rate telemetry. In Proceedings of the 2012 Annual International Conference of the IEEE Engineering in Medicine and Biology Society (EMBC), San Diego, CA, USA, 28 August-1 September 2012; IEEE: Piscataway, NJ, USA, 2012; pp. 3535-3538.

2. Liu, T.; Anders, J.; Ortmanns, M. System level model for transcutaneous optical telemetric link. In Proceedings of the IEEE International Symposium on Circuits and Systems (ISCAS2013), Beijing, China, 19-23 May 2013; pp. 865-868.

3. Varotsos, G.K.; Nistazakis, H.E.; Aidinis, K.; Jaber, F.; Rahman, K.M. Transdermal Optical Wireless Links with Multiple Receivers in the Presence of Skin-Induced Attenuation and Pointing Errors. Computation 2019, 7, 33. [CrossRef]

4. Miranda, H.; Gilja, V.; Chestek, C.A.; Shenoy, K.V.; Meng, T.H. A High-Rate Long-Range Wireless Transmission System for Simultaneous Multichannel Neural Recording Application. IEEE Trans. Biomed. Circuits Syst. 2010, 4, 181-191. [CrossRef]

5. Gil, Y.; Rotter, N.; Arnon, S. Feasibility of retroreflective transdermal optical wireless communication. Appl. Opt. 2012, 51, 4232-4239. [CrossRef] [PubMed]

6. Kim, H.-J.; Hirayama, H.; Kim, S.; Han, K.J.; Zhang, R.; Choi, J.-W. Review of Near-Field Wireless Power and Communication for Biomedical Applications. IEEE Access 2017, 5, 21264-21285. [CrossRef]

7. Thompson, A.; Wade, S.; Pawsey, N.C.; Stoddart, P. Infrared Neural Stimulation: Influence of Stimulation Site Spacing and Repetition Rates on Heating. IEEE Trans. Biomed. Eng. 2013, 60, 3534-3541. [CrossRef] [PubMed]

8. Ryu, S.I.; Shenoy, K.V. Human cortical prostheses: Lost in translation? Neurosurg. Focus 2009, 27, E5. [CrossRef] [PubMed]

9. Trevlakis, S.E.; Boulogeorgos, A.-A.A.; Karagiannidis, G.K. Signal Quality Assessment for Transdermal Optical Wireless Communications under Pointing Errors. Technologies 2018, 6, 109. [CrossRef]

10. Trevlakis, S.E.; Boulogeorgos, A.-A.A.; Sofotasios, P.C.; Muhaidat, S.; Karagiannidis, G.K. Optical wireless cochlear implants. Biomed. Opt. Express 2019, 10, 707-730. [CrossRef] [PubMed]

11. Ghassemlooy, Z.; Arnon, S.; Uysal, M.; Xu, Z; Cheng, J. Emerging Optical Wireless Communications-Advances and Challenges. IEEE J. Sel. Areas Commun. 2015, 33, 1738-1749. [CrossRef]

12. Liu, T.; Anders, J.; Ortmanns, M. Bidirectional optical transcutaneous telemetric link for brain machine interface. Electron. Lett. 2015, 51, 1969-1971. [CrossRef]

13. Liu, X.; Zhu, H.; Qiu, T.; Sritharan, S.Y.; Ge, D.; Yang, S.; Zhang, M.; Richardson, A.G.; Lucas, T.H.; Engheta, N.; et al. A fully integrated sensor-brain-machine interface system for restoring somatosensation. IEEE Sens. J. 2020, 21, 4764-4775. [CrossRef]

14. Lim, J.; Moon, E.; Barrow, M.; Nason, S.R.; Patel, P.R.; Patil, P.G.; Oh, S.; Lee, I.; Kim, H.-S.; Sylvester, D.; et al. 26.9 A $0.19 \times$ $0.17 \mathrm{~mm} 2$ Wireless Neural Recording IC for Motor Prediction with Near-Infrared-Based Power and Data Telemetry. In Proceedings of the 2020 IEEE International Solid-State Circuits Conference, San Francisco, CA, USA, 16-20 February 2020; pp. 416-418.

15. Moon, E.; Barrow, M.; Lim, J.; Lee, J.; Nason, S.R.; Costello, J.; Kim, H.S.; Chestek, C.; Jang, T.; Blaauw, D.; et al. Bridging the "Last Millimeter" Gap of Brain-Machine Interfaces via Near-Infrared Wireless Power Transfer and Data Communications. ACS Photon. 2021, 8, 1430-1438. [CrossRef] [PubMed]

16. Farid, A.A.; Hranilovic, S. Outage Capacity Optimization for Free-Space Optical Links with Pointing Errors. J. Light. Technol. 2007, 25, 1702-1710. [CrossRef]

17. Varotsos, G.K.; Nistazakis, H.E.; Stassinakis, A.N.; Volos, C.K.; Christofilakis, V.; Tombras, G.S. Mixed Topology of DF Relayed Terrestrial Optical Wireless Links with Generalized Pointing Errors over Turbulence Channels. Technologies 2018, 6, 121. [CrossRef] 
18. Varotsos, G.K.; Nistazakis, H.E.; Stassinakis, A.N.; Tombras, G.; Christofilakis, V.; Volos, C.K. Outage performance of mixed, parallel and serial DF relayed FSO links over weak turbulence channels with nonzero boresight pointing errors. In Proceedings of the 2018 7th International Conference on Modern Circuits and Systems Technologies (MOCAST), Thessaloniki, Greece, 7-9 May 2018; pp. 1-4.

19. Varotsos, G.; Nistazakis, H.; Aidinis, K.; Roumelas, G.; Jaber, F.; Rahman, K. Modulated Retro-Reflector Transdermal Optical Wireless Communication Systems with Wavelength Diversity over Skin-Induced Attenuation and Pointing Errors. In Proceedings of the 2019 IEEE International Symposium on Signal Processing and Information Technology (ISSPIT), Ajman, United Arab Emirates, 10-12 December 2019; IEEE: Piscataway, NJ, USA, 2019; pp. 1-5.

20. Bashkatov, A.; Genina, E.; Kochubey, V.; Tuchin, V. Optical properties of human skin, subcutaneous and mucous tissues in the wavelength range from 400 to $2000 \mathrm{~nm}$. J. Phys. D Appl. Phys. 2005, 38, 2543-2555. [CrossRef]

21. Ding, H.; Lu, J.Q.; A Wooden, W.; Kragel, P.J.; Hu, X.-H. Refractive indices of human skin tissues at eight wavelengths and estimated dispersion relations between 300 and $1600 \mathrm{~nm}$. Phys. Med. Biol. 2006, 51, 1479-1489. [CrossRef]

22. Parmentier, S.; Fontaine, R.; Roy, Y. Laser diode used in $16 \mathrm{Mb} / \mathrm{s}, 10 \mathrm{~mW}$ optical transcutaneous telemetry system. In Proceedings of the Biomedical Circuits and Systems Conference, BioCAS, Baltimore, MD, USA, 20-22 November 2008; IEEE: Piscataway, NJ, USA, 2008; pp. 377-380.

23. Ritter, R.; Handwerker, J.; Liu, T.; Ortmanns, M. Telemetry for Implantable Medical Devices: Part 1-Media Properties and Standards. IEEE Solid State Circuits Mag. 2014, 6, 47-51. [CrossRef]

24. Varotsos, G.K.; Nistazakis, H.E.; Aidinis, K.; Jaber, F.; Rahman, K.K.M. Transdermal subcarrier L-PSK or DBPSK optical wireless links with time diversity, skin attenuation and spatial jitter. J. Mod. Opt. 2020, 67, 1233-1240. [CrossRef]

25. Chevalier, L.; Sahuguede, S.; Julien-Vergonjanne, A. Optical Wireless Links as an Alternative to Radio-Frequency for Medical Body Area Networks. IEEE J. Sel. Areas Commun. 2015, 33, 2002-2010. [CrossRef]

26. Song, Y.-K.; Patterson, W.R.; Bull, C.W.; Borton, D.A.; Li, Y.; Nurmikko, A.V.; Simeral, J.D.; Donoghue, J.P. A Brain Implantable Microsystem with Hybrid RF/IR Telemetry for Advanced Neuroengineering Applications. In Proceedings of the 2007 29th Annual International Conference of the IEEE Engineering in Medicine and Biology Society, Lyon, France, 22-26 August 2007; pp. 445-448.

27. Abita, J.L.; Schneider, W. Transdermal optical Communications; John Hopkins APL Tech: Laurel, MD, USA, 2004; Volume 25, pp. 261-268.

28. Liu, T.; Bihr, U.; Anders, J.; Ortmanns, M. Performance evaluation of a low power optical wireless link for biomedical data transfer. In Proceedings of the 2014 IEEE International Symposium on Circuits and Systems (ISCAS), Melbourne, VIC, Australia, 1-5 June 2014; pp. 870-873.

29. Liu, T.; Bihr, U.; Becker, J.; Anders, J.; Ortmanns, M. In vivo verification of a $100 \mathrm{Mbps}$ transcutaneous optical telemetric link. In Proceedings of the Biomedical Circuits and Systems Conference (BioCAS), Lausanne, Switzerland, 22-24 October 2014; IEEE: Piscataway, NJ, USA, 2014; pp. 580-583.

30. Abualhoul, M.Y.; Svenmarker, P.; Wang, Q.; Andersson, J.Y.; Johansson, A.J. Free space optical link for biomedical applications. In Proceedings of the 2012 Annual International Conference of the IEEE Engineering in Medicine and Biology Society, San Diego, CA, USA, 28 August-1 September 2012; IEEE: Piscataway, NJ, USA, 2012; pp. 1667-1670.

31. Ackermann, D.M.; Smith, B.; Kilgore, K.L.; Peckham, P.H. Design of a high speed transcutaneous optical telemetry link. In Proceedings of the 2006 International Conference of the IEEE Engineering in Medicine and Biology Society, New York, NY, USA, 30 August-3 September 2006; IEEE: Piscataway, NJ, USA, 2006; pp. 2932-2935.

32. Ackermann, D.M.; Smith, B.; Wang, X.-F.; Kilgore, K.; Peckham, P.H. Designing the Optical Interface of a Transcutaneous Optical Telemetry Link. IEEE Trans. Biomed. Eng. 2008, 55, 1365-1373. [CrossRef]

33. Okamoto, E.; Yamamoto, Y.; Inoue, Y.; Makino, T.; Mitamura, Y. Development of a bidirectional transcutaneous optical data transmission system for artificial hearts allowing long-distance data communication with low electric power consumption. $J$. Artif. Organs 2005, 8, 149-153. [CrossRef] [PubMed]

34. Trevlakis, S.E.; Boulogeorgos, A.A.A.; Karagiannidis, G.K. On the impact of misalignment fading in transdermal optical wireless communications. In Proceedings of the 7th International Conference on Modern Circuits and Systems Technologies (MOCAST), Thessaloniki, Greece, 7-9 May 2018; IEEE: Piscataway, NJ, USA, 2018; pp. 1-4.

35. Trevlakis, S.E.; Boulogeorgos, A.-A.A.; Karagiannidis, G.K. Outage Performance of Transdermal Optical Wireless Links in the Presence of Pointing Errors. In Proceedings of the 2018 IEEE 19th International Workshop on Signal Processing Advances in Wireless Communications (SPAWC), Kalamata, Greece, 25-28 June 2018; IEEE: Piscataway, NJ, USA, 2018; pp. 1-5.

36. Varotsos, G.K.; Nistazakis, H.E.; Tombras, G.S.; Aidinis, K.; Jaber, F.; Rahman, M. On the use of diversity in transdermal optical wireless links with nonzero boresight pointing errors for outage performance estimation. In Proceedings of the 20198th International Conference on Modern Circuits and Systems Technologies (MOCAST), Thessaloniki, Greece, 7-9 May 2019; IEEE: Piscataway, NJ, USA, 2019; pp. 1-4.

37. Varotsos, G.; Nistazakis, H.; Aidinis, K.; Jaber, F.; Nasor, M.; Rahman, K. Error Performance Estimation of Modulated Retroreflective Transdermal Optical Wireless Links with Diversity under Generalized Pointing Errors. Telecom 2021, 2, 167-180. [CrossRef] 
38. Varotsos, G.K.; Nistazakis, H.E.; Aidinis, K.; Jaber, F.; Nasor, M.; Rahman, K.K.M. On the Utilization of L-PAM Technique in Transdermal Optical Wireless Links with Stochastic Pointing Errors for ABER Performance Estimation. In Proceedings of the 2021 10th International Conference on Modern Circuits and Systems Technologies (MOCAST), Thessaloniki, Greece, 5-7 July 2021; pp. 1-4.

39. Varotsos, G.; Nistazakis, H.; Aidinis, K.; Jaber, F.; Rahman, K.K.M.; Tsigopoulos, A.; Christofilakis, V. Average BER Estimation of Retroreflective Transdermal Optical Wireless Links with Diversity, Attenuation and Spatial Jitter. In Proceedings of the 2020 9th International Conference on Modern Circuits and Systems Technologies (MOCAST), Bremen, Germany, 7-9 September 2020; IEEE: Piscataway, NJ, USA, 2020; pp. 1-4.

40. Varotsos, G.K.; Nistazakis, H.E.; Aidinis, K.; Jaber, F.; Rahman, K.K.M. Signal Intensity Estimation in Transdermal Optical Wireless Links with Stochastic Pointing Errors Effect. Technologies 2020, 8, 60. [CrossRef]

41. Anastasov, J.A.; Zdravković, N.M.; Djordjevic, G.T. Outage capacity evaluation of extended generalized-K fading channel in the presence of random blockage. J. Frankl. Inst. 2015, 352, 4610-4623. [CrossRef]

42. Stassinakis, A.N.; Nistazakis, H.E.; Varotsos, G.K.; Tombras, G.S.; Tsigopoulos, A.D.; Christofilakis, V. Outage capacity estimation of FSO links with pointing errors over gamma turbulence channels. In Proceedings of the 2016 5th International Conference on Modern Circuits and Systems Technologies (MOCAST), Thessaloniki, Greece, 12-14 May 2016; pp. 1-4.

43. Djordjevic, G.T.; Petkovic, M.; Spasic, M.; Antic, D.S. Outage capacity of FSO link with pointing errors and link blockage. Opt. Express 2016, 24, 219-230. [CrossRef] [PubMed]

44. Furrer, S.; Coronel, P.; Dahlhaus, D. Simple ergodic and outage capacity expressions for correlated diversity ricean fading channels. IEEE Trans. Wirel. Commun. 2006, 5, 1606-1609. [CrossRef]

45. Nistazakis, H.E.; Tombras, G.; Tsigopoulos, A.D.; Karagianni, E.A.; Fafalios, M.E. Capacity estimation of optical wireless communication systems over moderate to strong turbulence channels. J. Commun. Networks 2009, 11, 384-389. [CrossRef]

46. Gradshteyn, I.S.; Ryzhik, I.M. Table of Integrals, Series, and Products, 6th ed.; Academic: New York, NY, USA, 2000.

47. Helstrom, C.W. Probability and Stochastic Processes for Engineers; Macmillan Coll Division: New York, NY, USA, 1991.

48. Maxim Integrated Products. 155 Mbps Low-Noise Transimpedance Amplifier. Available online: http:/ / pdf.datasheetcatalog. com/datasheets2/44/444242_1.pdf (accessed on 29 August 2021). 